\title{
Nonlinear electronic transport and enhanced catalytic behavior caused by native oxides on $\mathrm{Cu}$ nanowires
}

\author{
Rashad Hajimammadov, Zita Csendes, Juha-Matti Ojakoski, Gabriela Simone Lorite, Melinda Mohl, \\ and Krisztian Kordas ${ }^{\dagger}$
}

Microelectronics Research Unit, Faculty of Information Technology and Electrical Engineering, University of Oulu, P.O. Box 4500, 90570 Oulu, Finland

† Corresponding author, email: lapy@ee.oulu.fi

\begin{abstract}
Electrical transport properties of individual nanowires (both in axial and transversal directions) and their random networks suggest rapid oxidation when $\mathrm{Cu}$ is exposed to ambient conditions. The oxidation process is elucidated by thorough XRD, XPS and Raman analyzes conducted for a period of 30 days. Based on the obtained experimental data, we may conclude that first, cuprous oxide and copper hydroxide form that finally transform to cupric oxide. In electrical applications, oxidation of copper is not a true problem as long as thin films or bulk metal is concerned. However, as highlighted in our work, this is not the case for nanowires, since the oxidized surface plays quite important role in the contact formation and also in the conduction of percolated nanowire networks. On the other hand, by taking advantage of the mixed surface oxide states present on the nanowires along with their large specific surface area, we tested and found excellent catalytic activity of the oxidized nanowires in phenol oxidation, which suggests further applications of these materials in catalysis.
\end{abstract}

\section{Keywords}

$\mathrm{Cu}$ nanowires; oxidation; electrical transport; surface oxide; catalytic activity 


\section{Introduction}

The growing interest towards novel nanomaterials to replace costly noble metals such as gold, platinum, and palladium in various electrical applications has fostered considerable effort during the past years. Among the most promising materials, copper nanowires attracted a special attention due to its low cost compared to noble metals, and to the relatively simple synthesis even in large quantities. Owing to the superior electrical conductivity of metallic copper, buried micro- and nanostructures of the metal have been used in microprocessors (Damascene process) for nearly 20 years to replace aluminium interconnects ${ }^{1}$. With the recent development of lithography-free production methods of copper nanowires e.g. by solvothermal synthesis, ${ }^{2-5}$ electrochemical deposition in templates $^{6,7}$, solid state electrochemical reduction ${ }^{8}$ as well as by chemical reduction of oxide nanowires ${ }^{9}$, new perspectives for the applications of low dimensional $\mathrm{Cu}$ nanostructures are expected in the near future. So far, the practical applications are limited to surface-enhanced Raman scattering ${ }^{10}$, electrochemical capacitors ${ }^{11}$ and infrared polarizers $^{12}$ for pure $\mathrm{Cu}$ nanowires. Random percolated networks of ultra-long nanowires subjected to annealing at moderate temperatures have been shown as stable electrical wirings in transparent conductive electrodes ${ }^{13}$.

In spite of the numerous advantages that $\mathrm{Cu}$ may offer in many applications, the use of its nanostructures in ordinary conditions may pose challenges as it does not develop a thin natural continuous oxide layer on its surface (unlike e.g. lead, aluminum and titanium) that could efficiently protect the metal from corrosion. In order to prevent from oxidation, $\mathrm{Cu}$ nanowires are typically coated with or embedded in inorganic materials as well as polymers thus retaining the metallic properties of $\mathrm{Cu}$ in core-shell or composite nanostructures. For instance, $\mathrm{SiO}_{2}$ coated $\mathrm{Cu}$ core-shell nanowires were demonstrated in surface-enhanced Raman scattering ${ }^{14}$, while graphene encapsulated nanowires ${ }^{15}$ and reduced graphene oxide laminated nanowire films ${ }^{16}$ were used as transparent electrodes. Composites with PEDOT:PSS or with elastic poly(acrylate) matrix offer another approach for obtaining stable transparent electrodes e.g. for solar cells ${ }^{17}$ and flexible coatings ${ }^{18}$.

By reacting with oxygen from the atmosphere, copper forms two narrow band gap oxides such as cuprous oxide $\left(\mathrm{Cu}_{2} \mathrm{O}, 2.2 \mathrm{eV}\right)$ and cupric oxide $(\mathrm{CuO}, 1.4 \mathrm{eV})$. For practical reasons thus, it is important to reveal any changes in the chemical composition of the nanowires when exposed to air as the degree of oxidation and the oxide phases present in the material can significantly influence the utility of the nanowires. Metallic nanowires would be ideal for electrical interconnects and coatings as listed earlier, while nanoparticles, nanowires and thin films of 
semiconducting oxide phases may find their applications in liquid crystal displays ${ }^{19}$, catalysis ${ }^{20,21}$, field-effect transistors ${ }^{22}$ but also in solar cells ${ }^{23}$.

The oxidation of bulk and thin film copper under various conditions are extensively studied ${ }^{24-27}$, and also some reports on the oxidation of copper nanowires is available in the open literature ${ }^{28-30}$ however no systematic measurements are made yet for any thorough explication of electrical behavior and its relation to possible surface oxidation phenomena. Thus, in this work, we study the electrical properties of individual nanowires as well as random networks of $\mathrm{Cu}$ in detail, and apply a number of different surface and structure characterization techniques to elaborate the nature and progress of the surface oxidation under atmospheric conditions. Furthermore, we propose the oxidized $\mathrm{Cu}$ nanowires to be used as novel efficient heterogeneous catalytic nanomaterials.

\section{Materials and Methods}

\section{Synthesis of copper nanowires}

Copper nanowires were synthesized by a hydrothermal method ${ }^{3}$. Copper chloride dihydrate $\mathrm{CuCl}_{2} \cdot 2 \mathrm{H}_{2} \mathrm{O}$, Dglucose and 1-hexadecylamine (Sigma Aldrich) were used as received without further purification. Copper chloride dihydrate $(0.17 \mathrm{mg})$ was dissolved in deionized water $(80 \mathrm{~mL})$ then D-glucose $(0.39 \mathrm{mg})$ and hexadecylamine $(1.44$ $\mathrm{mg}$ ) were added slowly under vigorous stirring. After 5 hours stirring a light blue turbid dispersion formed and was placed into a Teflon ${ }^{\circledR}$ lined autoclave (Parr Instrument, Model 4525, $1000 \mathrm{~mL}$ ) and heated to $393 \mathrm{~K}$. After 24 hours of reaction and spontaneous cooling, the obtained brown dispersion was collected, centrifuged at $2500 \mathrm{rpm}$ (990 RCF) for 15 min. The supernatant was discarded and the reddish solid was redispersed in DI water, ethanol and $\mathrm{n}$-hexane to clean the product. The centrifuging, decantation and redispersion cycle was repeated three times in each solvent and finally the obtained copper nanowires were kept under $n$-hexane until further analyses in order to prevent them from oxidation.

\section{Structural and surface analyses}

Field emission scanning electron microscopy (FESEM, Zeiss Ultra Plus) as well as transmission electron microscopy equipped with energy dispersive X-ray spectroscopy (JEOL JEM-2200FS EFTEM/EDX) were used for analyzing microstructure, morphology and elemental composition, respectively. X-ray diffraction (XRD) patterns were collected with a Siemens D5000 diffractometer ( $\mathrm{Cu} \mathrm{K} \alpha \lambda=1.542 \AA)$. Raman spectroscopy 
measurements were carried out using a Horiba Jobin-Yvon LABRAM HR800 UV-VIS $\mu$-Raman device with 488 nm Ar laser. X-ray photoelectron spectroscopy measurements were performed using a Thermo Fisher Scientific Escalab 250 XI XPS system (Al K $\alpha$ X-Ray source, $1486.6 \mathrm{eV}$, data evaluation suing Avantage software).

For XRD and XPS analyses the nanowires were drop casted from a hexane dispersion on the surface of a silicon wafer having (100) orientation and on microscope glass slide, respectively.

\section{Electrical characterization}

Two probe electrical measurements were performed on drop casted nanowires from hexane dispersion on Si chips $\left(1.5 \mu \mathrm{m}\right.$ thermal $\mathrm{SiO}_{2}$ with finger electrodes of sputtered $40 \mathrm{~nm}$ Ti and $\left.250 \mathrm{~nm} \mathrm{Pt}\right)$. Keithley system sourcemeter (2636A) was applied to source voltage and measure current on the test chips probed with a Wentworth Labs station. Temperature dependent electrical measurements were carried out in a computer controlled Linkam THMS600 heating stage using a Keithley 2612 system sourcemeter in Ar flow.

Atomic force microscopy (AFM, Multimode 8/ PF-TUNA, Nanoscope V, Bruker, USA) was used to evaluate the current-voltage (I-V) characteristics at the Cu nanowire surface. AFM was operated in spectroscopy mode in air using conductive Pt-coated conical Si probe with spring constant of $2.67 \mathrm{~N} / \mathrm{m}$ (calibrate via ThermalTune) and typical radius of $30 \mathrm{~nm}$ (NSC18/Pt MicroMash, Tallin, Estonia). Cu nanowires dispersed in hexane were drop casted on conductive Au-coated substrate. The substrate was mounted with a conductive carbon tape on AFM metal discs, and to ensure proper grounding, the gold surface was also connected with the AFM sample holder using silver paste. After topography imaging, a 20 points-line was selected on top of the Cu nanowire and the voltage was ramped up and down in the range from $-3 \mathrm{~V}$ to $+3 \mathrm{~V}$ while the current signal was recorded. The ramp was obtained three times each point. The same protocol was used on the gold-coated surface next to the $\mathrm{Cu}-$ nanowire to be used as reference. The measurements were repeated at least 3 to 5 times per day and the measurements were carried out for four consecutive days (day 0 to day 3 ) in the same nanowire. An average of the I-V ramp from 60 to 120 curves was obtained by using OriginPro software (OriginLab Corporation, USA). It has been noticed that in some cases (less than $20 \%$ of the total curves) the I-V ramp on top of the Cu nanowire looked like the gold-coated I-V ramp or the other extreme, no current was measured at all. This can be explained by the probe being misaligned compared to the previous image scan. Such obviously erroneous I-V ramps were excluded from the average. 


\section{Testing the catalytic activity}

The catalytic behavior of oxidized copper nanowires was tested in the wet air oxidation of phenol. The reaction was performed in a Teflon lined autoclave with a capacity of $1 \mathrm{~L}$. The reactor was filled with $180 \mathrm{~mL}$ DI water and loaded with $70 \mathrm{mg}$ of copper nanowires oxidized in a lab ambient at room temperature for 14 days. After adding $20 \mathrm{~mL}$ of a $50 \mathrm{~g} / \mathrm{L}$ phenol solution the reactor was closed and purged with synthetic air for $1 \mathrm{~h}$ in order to remove the contaminants which could affect the reaction. The final concentration of the phenol in the reaction mixture was $5 \mathrm{~g} / \mathrm{L}$. When the system reached its working temperature $413 \mathrm{~K}$, the pressure was $1.8 \times 10^{3} \mathrm{kPa}$ and the reaction started. The reaction time was $5 \mathrm{~h}$. Samples were withdrawn from the sampling valve each hour and the concentration of phenol was determined by a Hewlett-Packard 6890 GC system with HP-5973 MSD. The column used was a Chrompack CP WAX 52 CB $(30 \mathrm{~m} \times 0.25 \mathrm{~mm}, 0.25 \mu \mathrm{m}$ film). Since the oxidation of phenol results in its degradation to $\mathrm{CO}_{2}$ and water, the evolved gas was bubbled through a $11 \mathrm{~g} / \mathrm{L} \mathrm{Ba}(\mathrm{OH})_{2}$ solution in order to detect $\mathrm{CO}_{2}$ gravimetrically by collecting the formed $\mathrm{BaCO} 3$ precipitate. Each hour the precipitate was collected, filtered and dried for subsequent weighting and defining indirectly the amount of released $\mathrm{CO}_{2}$.

\section{Results and Discussion}

Scanning electron microscopy (Fig. 1) shows the formed nanowires are straight, have uniform diameter along the whole length, and the structure is free of any kinks or voids. Assessment of as synthesized nanowires by energydispersive $\mathrm{X}$-ray analysis indicates the nanowires are pure metallic $\mathrm{Cu}$, although a small amount of oxygen is present on the wire surface, which can be explained by exposure to atmospheric oxygen during sample preparation (drop casting on Ni grid from ethanol dispersion) and previous washing steps after the synthesis, where ethanol and water are used (which most probably contain dissolved oxygen). The average diameter is about $50 \mathrm{~nm}$ and the length of the nanowires was found to be in the range of $10-50 \mu \mathrm{m}$. Due to the large aspect ratio, nanowires in high density random networks tend to curve rather than break into smaller pieces caused by the mechanical stress. Besides the nanowires also nanoparticles with different shapes (tetrahedron, triangular pyramid, perfect cube and spherical form) and size are present in the product (Fig. 1). It is worth mentioning that the purification process has a significant effect on the particle shape distribution as the small nanoparticles undergo slower sedimentation than the nanowires during centrifugation thus, the supernatant fraction of the dispersion is always enriched in the 
small nanoparticles. Accordingly, it is possible to reduce and somewhat control the amount of nanoparticles in the product by simply repeating the purification cycles.

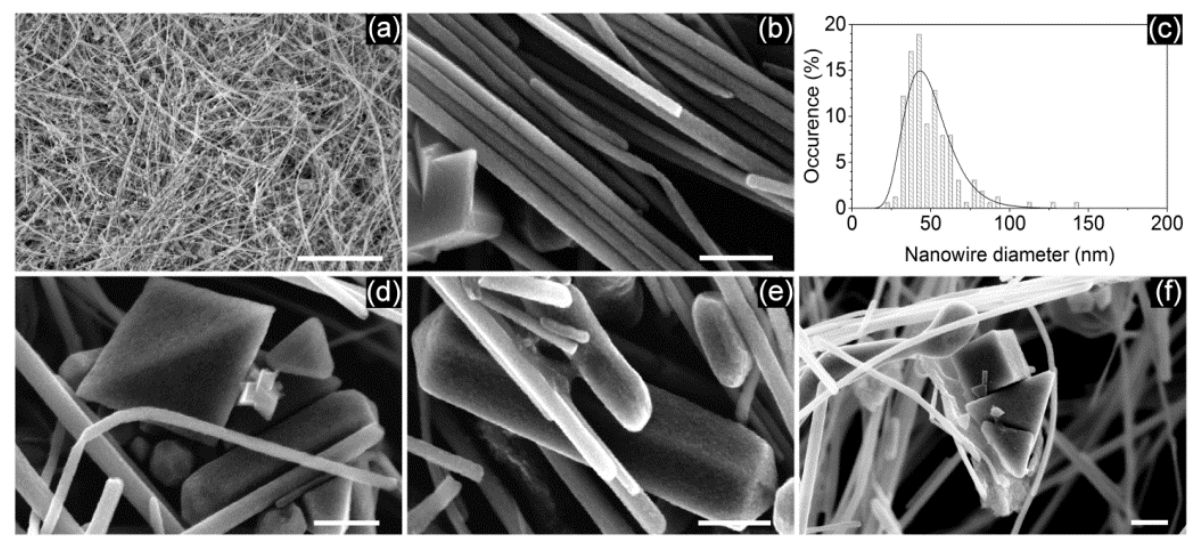

Figure $1(a, b)$ SEM images of the product and (c) size distribution of copper nanowires. The scale bars show $3 \mu \mathrm{m}$ and 200 $\mathrm{nm}$ respectively. SEM images of other crystalline products including (d) tetrahedron, truncated tetrahedron, truncated octahedron (e) nanorod with five-fold symmetry and (f) nanocube. Scale bars are $200 \mathrm{~nm}$.

For testing the electrical properties and its temporal change, which is of particular importance for practical use of these materials, random networks of the grown $\mathrm{Cu}$ nanowires were deposited between lithographically defined $\mathrm{Pt}$ microelectrodes on $\mathrm{Si} / \mathrm{SiO}_{2}$ chips. The resistance of the nanowire films were found to increase rapidly even after a few hours being stored in ambient air (Fig. 2 (a)). After a few days, the increase can be several orders of magnitude, which suggests the rapid development of semiconducting surface oxides acting as efficient barriers for electrons to inject from the electrodes to the nanowires and also from one nanowire to another in the percolated conduction path. The resistance of the nanowire networks stored in air (and then measured in argon flow) shows an exponential decrease with temperature, which is a clear signature of thermal activation typical for non-metallic conduction (Fig. 2(b)). The activation energy around room temperature is in the range between $0.4-0.5 \mathrm{eV}$ comparable with the barrier measured earlier for freshly deposited copper nanowire networks that developed oxides on their surface rapidly ${ }^{3}$. It is worth noting that the resistance of the nanowire network shows an anomaly in the first temperature scan. The resistance first starts to decrease on heating (as expected for the semiconducting copper oxide phases) then suddenly increases irreversibly with almost two orders of magnitudes after which, the temperature scan follows a reversible exponential change. The irreversible change around $400 \mathrm{~K}$ is not understood and cannot be explained by our measurements however might be associated with a recrystallization or structural rearrangement of the nanowires. 

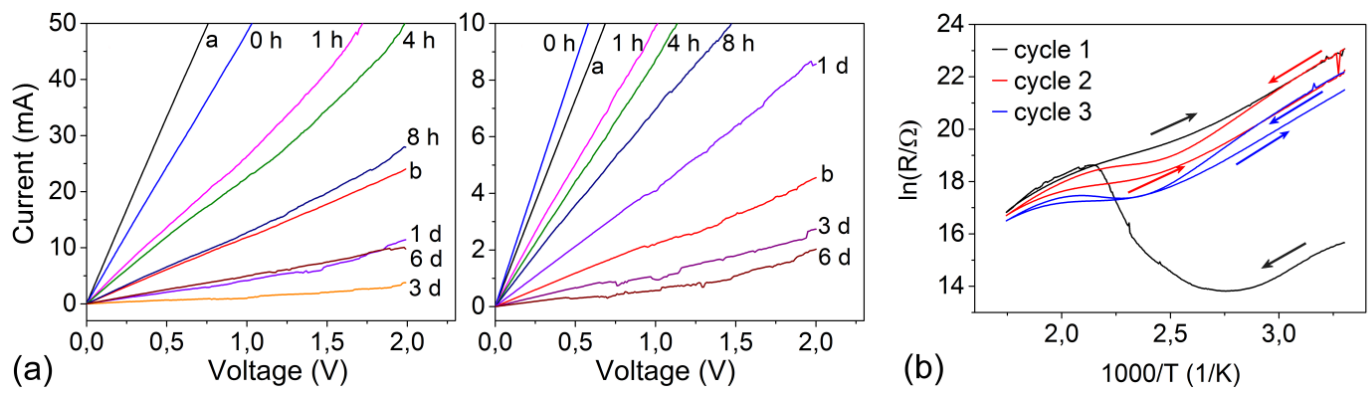

Figure 2 (a) Current-voltage plots for two similar Cu nanowire networks. Label "a" stands for measurements carried out in the glove box filled with $\mathrm{N}_{2}$ right after preparation, while label "b" denotes measurements of the corresponding chips stored in a glove box after 10 days. Plots labelled with 0 h, 1h, 4 h, 8 h, $1 \mathrm{~d}, 3 \mathrm{~d}$ and $6 \mathrm{~d}$ are measurement data acquired in ambient conditions (h and d mean hour and day, respectively) after removing the chips from the glove box. (b) Resistance vs. temperature curve of an oxidized $\mathrm{Cu}$ nanowire network measured between 303 and $573 \mathrm{~K}$ in argon.

Current-voltage measurements on individual nanowires (Fig. 3(a)) drop casted on Si chips with Pt electrodes display very similar behaviour as found for random networks of the nanowires. Right after the deposition between the electrodes ( 0 hour) the device showed nonlinear behaviour. The nonlinearity of the device is not caused by self-heating as the slope of the curve is higher at higher voltages. The measured I-V curves within the time period of 2 hours did not vary significantly, however 1 day exposure of the sample to ambient air caused a significant decrease of electrical conductivity similar to that reported earlier for template grown Cu nanowires with bottomside contacts. ${ }^{30}$ We assume, the continuously growing oxide layers on the surface of the nanowire results in increased barrier for the electrons to pass the Schottky interface between platinum electrode and the semiconducting copper oxide contact.

We have performed current-voltage on individual nanowires also in the transversal direction as shown in Fig. 3(b) by using atomic force microscopy (AFM) in spectroscopy mode. The nanowires in this case were drop casted on a gold-coated surface instead of a patterned chip and probed in 20 different locations (on the same wire) for 3 days of measurement period. The collected I-V curves show very similar nonlinear behavior as measured previously along the wires suggesting that the surface of nanowire has developed a barrier type of oxide layer very rapidly after drop casting from its hexane dispersion. 

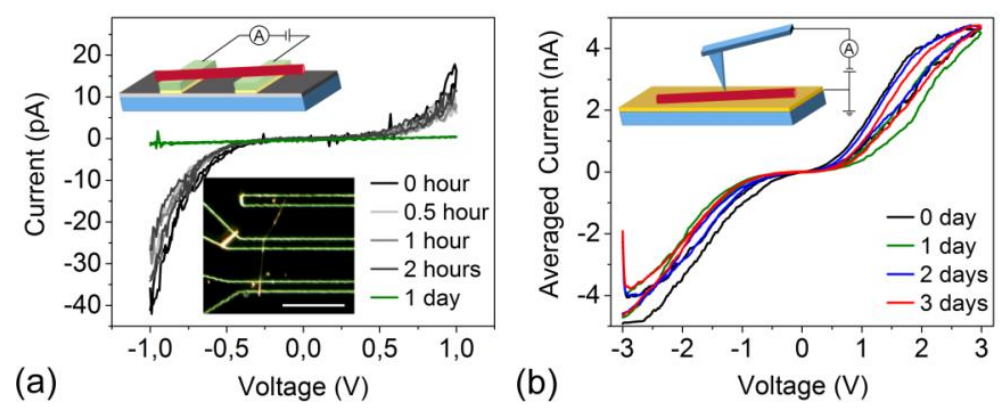

Figure 3 (a) Two probe current-voltage curves for an individual $\mathrm{Cu}$ nanowire undergoing oxidation in air. The inset shows the corresponding nanowire on the test chip observed by dark-field optical electron microscopy. The electrical measurement is carried out through the two outermost electrodes. Scale bar is $30 \mu \mathrm{m}$. (b) Current- voltage curves via AFM operated in spectroscopy mode for a single copper nanowire drop casted on a gold-coated surface. In this case, the Pt-coated AFM probe is positioned perpendicular to the nanowire axis.

To support our assumptions on the fast development of surface oxides, which eventually limit the electron transport in individual nanowires and also at the boundaries of several nanowires that are in contact, we have carried out a number of bulk and surface characterization measurements as a function of time.

For analyzing any crystalline phase evolution on the nanowires, X-ray powder diffraction was carried out on drop casted films. Reflections appearing in the XRD pattern at $43.4^{\circ}, 50.5^{\circ}, 74.1^{\circ}, 90.0^{\circ}$ and $95.2^{\circ}$ can be perfectly indexed to fcc $\mathrm{Cu}$ (111), (200), (220), (311) and (222) crystal planes, respectively (Fig. 4a). No other peaks corresponding to cuprous or cupric oxide were observed even up to 7 days of storage of the samples in ambient conditions. After 10 days a new low intensity peak assigned to cuprous oxide appeared at $36.4^{\circ}$, but crystalline cupric oxide was not observed even after 21 days. Since copper is known to develop oxides on its surface, the lacking reflections in the XRD patterns clearly indicate that either the oxides are not crystalline or have extremely small crystal size making it hard to detect. ${ }^{31,32}$

To reveal any non-crystalline oxide phases, we also performed $\mu$-Raman analysis. Spectra of the nanowires were collected up to 30 days of oxidation periods (Fig. 4b). The spectrum for the 0 day sample (i.e. fresh sample with maximum 5 min exposure to air) displays several sharp peaks at 106, 143, $218 \mathrm{~cm}^{-1}$ as well as broadened ones at around 530 and $630 \mathrm{~cm}^{-1}$ that correspond to $\mathrm{Cu}_{2} \mathrm{O}$ according to experimental ${ }^{31,33-35}$ and theoretical ${ }^{36}$ data in the literature. The spectra obtained in the first 7 days of experiments are very similar to the one measured at day 0 . Only the overall intensity of the individual spectra recorded at different days show some variations, which is caused by the variation of surface locations probed by the Raman laser (viz. the rough sample surface reflects differently 
in different locations). On day 10, two new peaks appeared at around $293 \mathrm{~cm}^{-1}$ and $339 \mathrm{~cm}^{-1}$ along with a broad band around $1100 \mathrm{~cm}^{-1}$, that are characteristic for cupric oxide $\mathrm{CuO}^{31,32,37}$. In addition, the peak located at around $630 \mathrm{~cm}^{-1}$ (originally assigned to $\mathrm{Cu}_{2} \mathrm{O}$ ) became broader after 10 days due to the appearance of a peak at $624 \mathrm{~cm}^{-1}$ that can be attributed to $\mathrm{CuO}^{31,37}$.

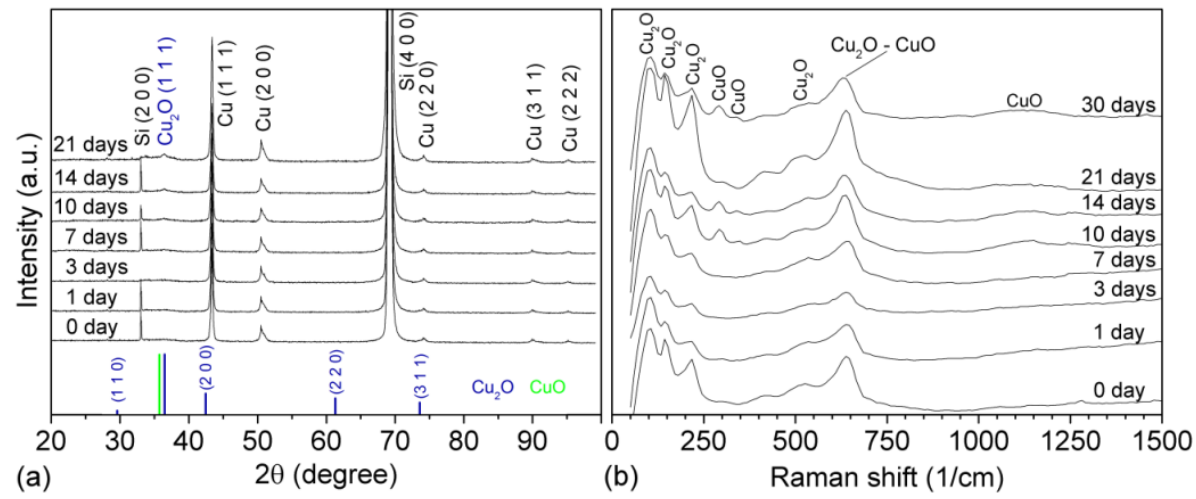

Figure 4 (a) X-ray diffraction patterns of copper nanowires and (b) Raman spectra of copper nanowires from 0-30 days. The $2 \theta$ angle positions of cupric oxide most intense peak (green) and cuprous oxide peaks (blue) are depicted. The samples were kept and oxidized in ambient laboratory air conditions (temperature of $21-23^{\circ} \mathrm{C}$ ). The relative humidity of air during storage was $\sim 70 \%$ (except for the sample measured by Raman spectroscopy, which was stored and analyzed in a clean room ISO 6 environment with controlled relative humidity of $\sim 43 \%$ ).

Qualitative assessment of copper oxidation states was achieved by high resolution Cu LMM Auger as well as Cu $2 \mathrm{p}_{3 / 2}$ and $\mathrm{O} 1 \mathrm{~s} \mathrm{X}$-ray photoelectron spectroscopy collected during the measurement period for up to 30 days (Fig. 5(a-c)). In order to compensate for any energy shifts (e.g. due to surface charging) the spectra are referenced to the main component in C 1s spectra (at $284.8 \mathrm{eV}$ ) recorded on each measurement day (Fig. 5(d)). According to the resolved peaks centered at $935.1 \mathrm{eV}\left(\mathrm{Cu}(\mathrm{OH})_{2}\right), 933.3 \mathrm{eV}(\mathrm{CuO}), 932.2\left(\mathrm{Cu}_{2} \mathrm{O}\right.$ and/or $\left.\mathrm{Cu}\right)$ and $931.1 \mathrm{eV}$ (unidentified) in the $\mathrm{Cu} 2 \mathrm{p}_{3 / 2}$ spectra a number of different products were found to be present on the surface from the beginning of the analysis. Already at the 0 day the presence of low intensity $\mathrm{CuO}$ and $\mathrm{Cu}(\mathrm{OH})_{2}$ peaks is somewhat surprising, since the mechanism of $\mathrm{Cu}$ oxidation is believed to proceed first with the formation of $\mathrm{Cu}_{2} \mathrm{O}$ phase, which subsequently transforms to hydroxide and cupric oxide ${ }^{38}$.

The immediate detection of hydroxide may be due to the wet chemical synthesis as well as to the washing step in water $^{38}$ (besides hexane and ethanol). However, the rising intensity of the $\mathrm{Cu}(\mathrm{OH})_{2}$ peak after day 0 indicates that the surface absorption of water vapor from the atmosphere is significant upon storage in ambient conditions. Dehydration and transformation of the metastable hydroxide phase to cupric oxide seems to occur simultaneously 
according to the immediate appearance of the low intensity $\mathrm{CuO}$ peak at around $933.3 \mathrm{eV}$ as well as the corresponding low intensity satellites between 943 and $947 \mathrm{eV} \cdot{ }^{39}$ Although the intensity of these satellites varies depending on the day of the measurement, they stay rather low during the entire duration of our experiments, i.e. the overall amount of the $\mathrm{CuO}$ phase on the surface is very small compared to $\mathrm{Cu}_{2} \mathrm{O}$ or $\mathrm{Cu}$ phases. As there is only a little difference in binding energies of metallic $\mathrm{Cu}$ and $\mathrm{Cu}_{2} \mathrm{O}$, these two components cannot be distinguished from each other in the $\mathrm{Cu} 2 \mathrm{p}_{3 / 2}$ spectrum thus we analyzed these components by Auger spectroscopy.

The results of Cu LMM Auger spectra depicted in Fig. 5b, show that on day 0 both metallic and oxide states of copper are present at the same time. This proves that the time period between the preparation of the sample and the measurement was not enough for the oxidation of the whole surface (or at least not deeper than a few nanometers). In fact, for $\mathrm{CuO}$ and $\mathrm{Cu}(\mathrm{OH})_{2}$ the same trend is observed here as on the $\mathrm{Cu} 2 \mathrm{p}_{3 / 2}$ spectra though, here the $\mathrm{Cu}_{2} \mathrm{O}$ phase can be distinguished clearly from the metallic $\mathrm{Cu}$ component by the resolved peaks at 916.8 $\mathrm{eV}$ and $918.6 \mathrm{eV}$, respectively. Extended period of exposure to oxygen resulted in the rise of cupric oxide peak at $917.7 \mathrm{eV}$, whereas the metallic $\mathrm{Cu}$ peak at $918.6 \mathrm{eV}$ is gradually decreasing and disappearing by day 21 of storage in air. The continuous oxidation of the nanowires can be well explained by the good diffusion of Cu through the oxides $^{40}$, while the increasing amount of $\mathrm{Cu}(\mathrm{OH})_{2}$ is due to the adsorbed water from the atmosphere reacting with the surface oxides. The broad feature in the Cu LMM spectra at around $913 \mathrm{eV}$ is related with the Coster-Kronig transition $^{41}$.

Analysis of $\mathrm{O}$ 1s spectra (Figure 5c) supports the previous observations related to the change in the oxidation states of copper. The measurement performed on the 0 day shows a high intensity peak located at $530.1 \mathrm{eV}$ that confirms the presence $\mathrm{Cu}_{2} \mathrm{O}$. The other two peaks with significantly lower intensities located at $529.2 \mathrm{eV}$ and at $530.5 \mathrm{eV}$ correspond to $\mathrm{CuO}$ and $\mathrm{Cu}(\mathrm{OH})_{2}$, respectively. The intensities of the $\mathrm{CuO}$ and $\mathrm{Cu}(\mathrm{OH})_{2}$ peaks continuously increase with the storage time. In addition, a fourth broad peak gradually appears around $532.5 \mathrm{eV}$ related to organic compounds adsorbed from air. As for the C 1s core level component, the main peak at 284.8 $\mathrm{eV}$ is associated with adsorbed organics having $\mathrm{C}-\mathrm{C}$ bonds. A minor peak at $283.3 \mathrm{eV}$ suggests metal carbide ${ }^{42,43}$ on the surface, which may explain the low intensity unidentified component in the $\mathrm{Cu} 2 \mathrm{p}_{3 / 2}$ spectrum at $931.1 \mathrm{eV}$. The existence of carbide peak at $283.3 \mathrm{eV}$ may be explained by the interaction of hexane (storage medium before the oxidation experiments). Other minor peaks also found at higher energies (286-288 eV) are associated with 
oxygenates having $\mathrm{C}-\mathrm{O}, \mathrm{C}=\mathrm{O}$ and $\mathrm{O}=\mathrm{C}-\mathrm{O}$ bonds, ${ }^{25}$ however surface sulfates and carbonates that can potentially form from the atmospheric impurities upon extended period of exposure were not detected.

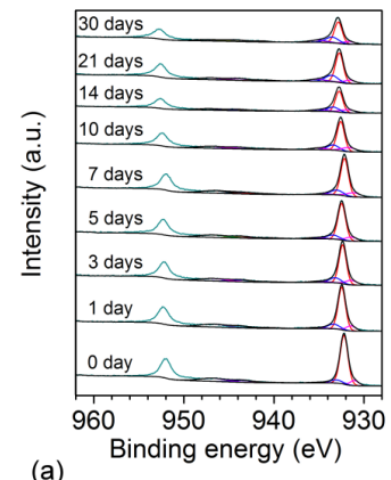

(a)

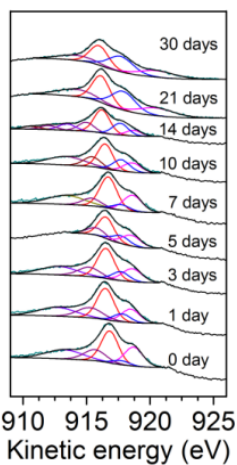

(b)

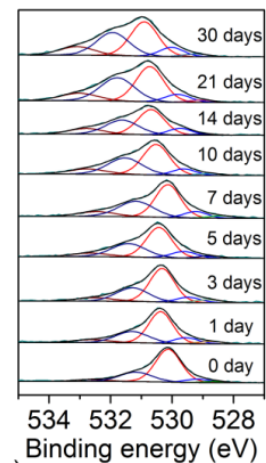

(c)

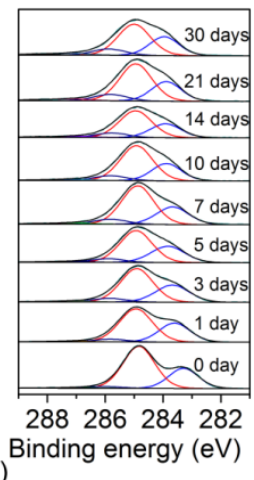

(d)

Figure $5 \mathrm{X}$-ray photoelectron spectra of $\mathrm{Cu}$ nanowires resolving the (a) $\mathrm{Cu}$ 2p $\mathrm{p}_{3 / 2}$, (b) Cu LMM Auger, (c) O 1s and (d) C 1s peaks.

Based on the assessment of XPS data thus the evolution of copper oxidation starts by the simultaneous formation of cuprous oxide and hydroxide, which then oxidize further to cupric oxide. The progress of oxidation is well illustrated by the change of concentration of various components in the nanowire powder (Fig. 6).
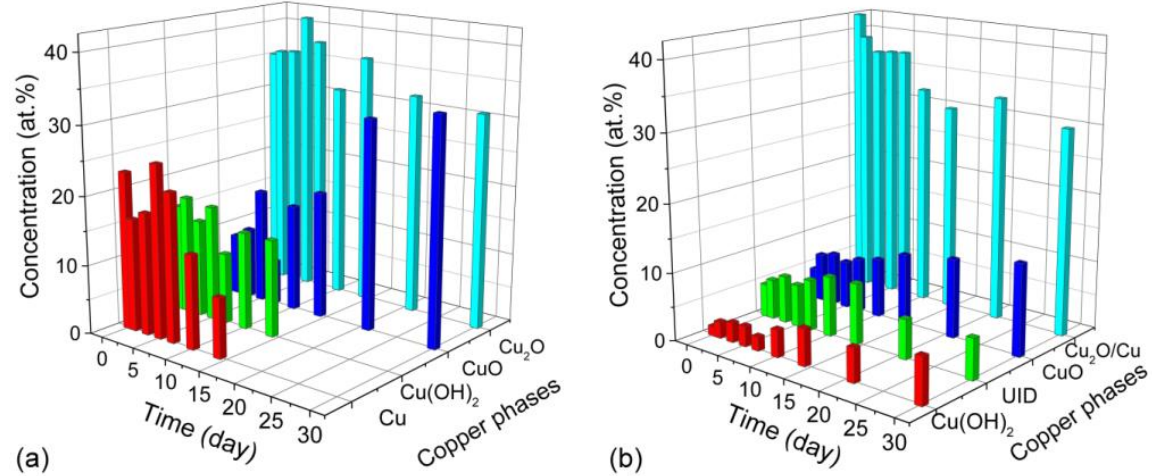

Figure 6 Temporal evolution of copper oxidation by following up the concentration of metal, oxide and hydroxide phases by evaluating the (a) Cu LMM Auger and (b) $\mathrm{Cu} 2 \mathrm{p}_{3 / 2}$ spectra.

Although the partial oxidation of these hydrothermally grown $\mathrm{Cu}$ nanowires seems to hinder direct applications of these materials in electronics, the coexistence of various oxide phases may be beneficial for surface mediated redox processes, i.e. for heterogeneous chemical reactions $s^{44-46}$ as well as for electrochemical applications $s^{47-50}$. To check whether the oxidized nanowires studied in this work are suitable as catalyst, here we select a simple model reaction, namely the catalytic phenol oxidation with wet air. Nanowires of oxidized $\mathrm{Cu}$ were dispersed in aqueous phenol solution and air was then bubbled through in an autoclave heated to $413 \mathrm{~K}$. According to gaschromatography measurements, the nanowires are catalytically highly active as indicated by the quick degradation 
of phenol ( $1 \mathrm{~g}$ in $200 \mathrm{~mL}$ water) over only $70 \mathrm{mg}$ of catalyst within 5 hours of reaction. Phenol conversion measured by gas chromatography was also compared to values obtained indirectly from $\mathrm{BaCO}_{3}$ formation (by bubbling the downstream products through $\mathrm{Ba}(\mathrm{OH})_{2}$ solution). The results show considerable difference of the two conversion values indicating that a large fraction of phenol $(\sim 70 \%)$ is not mineralized (i.e. oxidized to water and $\left.\mathrm{CO}_{2}\right)$ and possibly forming also other products and decomposition intermediates. Pires ${ }^{51}$ et al. performed a similar process testing the catalytic activity of different materials including commercial $\mathrm{CuO}$. According to their report, the overall catalytic performance of $\mathrm{CuO}$ is quite comparable to other copper containing catalysts on different supports, although with pronounced formation of acetic acid in the product besides $\mathrm{CO}_{2}$. Interestingly, in our case we could not identify any acetic acid or other byproducts in the reaction mixture with GC-MS suggesting the formation of some insoluble gas products and/or solid precipitates. After the reaction thus the reactant mixture in the vessel was filtered and dried, and the collected powder was then analyzed by SEM, which revealed polyphenol nanospheres having size of about $200 \mathrm{~nm}$ in the products (inset in Fig. 7). Earlier studies briefly indicated the possibility of phenol polymerization as a result of oxidation reaction in aqueous media ${ }^{52,53}$. Some authors used stabilizers to polymerize phenol and their reports showed polyphenol nanospheres very similar to our results ${ }^{54,55}$. In here, we could prepare polyphenol nanoparticles without the addition of any stabilizer. The adsorption of the polymeric product ${ }^{56}$ on the surface of catalyst with subsequent decreasing of activity was reported for CuO catalyst, which coincides with our results.

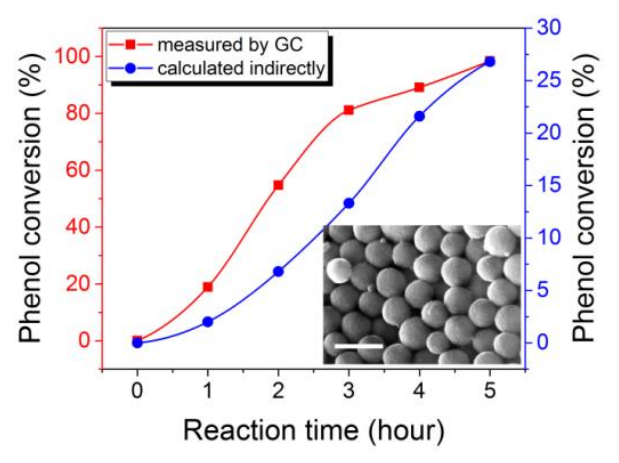

Figure 7 Phenol conversion as a function of reaction time, measured by GC (red line) and calculated indirectly from the weight of $\mathrm{BaCO}_{3}$ (blue line). In the inset, a SEM image of polymerized phenol nanoparticles is shown (scale bar is $500 \mathrm{~nm}$ ). 


\section{Conclusions}

Electrical measurement data on individual $\mathrm{Cu}$ nanowires and on random networks indicate a dramatic decrease of electrical conductance after a few days of storage. According to X-ray diffraction as well as $\mu$-Raman, X-ray photoelectron and Auger spectroscopy, $\mathrm{Cu}$ nanowires synthesized by the hydrothermal route undergo rapid oxidation when stored in ambient conditions similar to other $\mathrm{Cu}$ surfaces made by different methods. The main oxide phase is $\mathrm{Cu}_{2} \mathrm{O}$, however both $\mathrm{CuO}$ and $\mathrm{Cu}(\mathrm{OH})_{2}$ are gradually forming on the surface. Storage for weeks in air eventually results in the complete loss of metallic copper on the surface. According to the results of this work, the poor electrical interface between the nanowires is caused by the formed semiconducting phases on the surface. Based on the data presented here, direct applications of the hydrothermally grown nanowires in electronics is limited, however the mixed surface oxide states may be very valuable e.g. in heterogeneous catalysis as active surfaces in redox reactions.

\section{References}

1. Andricacos C., Uzoh C., Dukovic, J. Horkans J. O., Deligianni H. IBM J. Res. Dev. 42, 567-574, (1998).

2. Asano Y., Nakaoka K., Murashiro K., Komatsu T., Hoshino K. Langmuir 21, 3746-3748 (2005).

3. Mohl M., Pusztai P., Kukovecz A., Konya Z., Kukkola J., Kordas K., Vajtai R., Ajayan P. M. Langmuir 26 (21), 16496-16502 (2010).

4. Jiang Z., Tian Y., Ding S. Mater. Lett. 136, 310-313 (2014).

5. Zhao Y., Zhang Y., Li Y., He Z., Yan Z. RSC Adv. 2, 11544-11551 (2012).

6. Molares T. M. E., Buschmann V., Dobrev D., Neumann R., Scholz R., Schuchert I. U., Vetter J. Adv. Mater. 13, 62-65 (2001).

7. YiTian P., QuanFang Ch. Chin. Sci. Bull. 58, 3409-3414 (2013).

8. $\quad$ Xu D., Dong Z., Sun J.L. Mater. Lett. 92,143-146 (2013).

9. Han J.W., Meyyappan M. US Patent, 8834597 (2014). 
10. Yao J. L,. Pan G. P, Xue K. H., Wu D. Y., Ren B., Sun D. M., Tang J., Xu X., Tian, Z. Q. Pure Appl. Chem. 72, 221-228 (2000).

11. Asano Y., Nakaoka K., Murashir K., Komatsu T., Hoshino K. Mater. Lett. 81, 162-164 (2012).

12. Pang Y.T., Meng G.W., Zhang Y., Fang Q., Zhang L.D. Appl. Phys. A. 76, 533-536 (2003).

13. Guo H., Lin N., Chen Y., Wang Z., Xie Q., Zheng T., Gao N., Li Sh., Kang J., Cai D., Peng D.L. Sci. Rep. 3, 2323 (2013).

14. Pang Ch., Cui H., Wang Ch. RSC Adv. 4, 31887-31891 (2014).

15. Mehta R., Chugh S., Chen Z.H. Nano Lett. 15, 2024-2030 (2015).

16. Kholmanov I. N., Domingues S. H., Chou H., Wang X., Tan Ch., Kim J.Y., Li H., Piner R., Zarbin A. J. G., Ruoff R. S. ACS Nano 7, 1811-1816 (2013).

17. Chen J.Y., Zhou W.X., Chen J., Fan Y., Zhang Z.Q., Huang Z.D., Feng X.M., Mi B.X., Ma Y.W., Huang W. Nano Res. 8, 1017-1025 (2015).

18. Cheng Y., Wang Sh., Wang R., Sun J., Gao L. J. Mater. Chem. C. 2, 5309-5316 (2014).

19. Malik A., Prakash J., Kumar A., Dhar A., Biradar A. M. J. Appl. Phys. 112, 054309-054309 (2012).

20. Poreddy R., Engelbrekt C., Riisager A. Catal. Sci. Technol. 5, 2467-2477 (2015).

21. Jing L., Zailei Z., Yongjun J., Zheying J., Shanying Z., Ziyi Z., Fabing S. Nano Res. 9(5), 1377-1392 (2016).

22. Vaseem M., Hong A.R., Kim R.T., Hahn Y.B. J. Mater. Chem. C. 1, 2112-2120 (2013).

23. Fujimoto K., Oku T., Akiyama T., Suzuki A. J. Phys. 433, 012024 (2013).

24. Park J., Lim K., Ramsier R. D., Kang Y.Ch. Bull. Korean Chem. Soc. 32, 3395-3399 (2011).

25. Cano E., Torres C.L., Bastidas J. M. Mater. Corrosion. 52, 667-676 (2001).

26. Suzuki S., Ishikawa Y., Isshiki M., Waseda Y. Mater. Trans., JIM 38(11), 1004-1009 (1997).

27. Iijima J., Lim J.-W., Hong S.-H., Suzuki S., Mimura K., Isshiki M. Appl. Surf. Sci. 253, 2825-2829 (2006).

28. Sun J.-L., Xu J., Zhu J.-L. Appl. Phys. Lett. 90, 201119 (2007).

29. Liu Z., Bando Y. Chem. Phys. Lett. 378, 85-88 (2003). 
30. Toimil Molares M. E., Höhberger E. M., Schaeflein Ch., Blick R. H., Neumann R., Trautmann C. Appl. Phys. Lett. 82, 2139 (2003).

31. Gong Y.S., Lee Ch., Yang C. K. J. Appl. Phys. 77, 5422-5425 (1995).

32. Gan Z.H., Yu G.Q., Tay B.K., Tan C.M., Zhao Z.W., Fu Y.Q. J. Phys. D: Appl. Phys. 37, 81-85 (2004).

33. Mayer S. T., Muller R. H. J. Electrochem. Soc. 139, 426-434 (1992).

34. Wang W., Tu Y., Wang L., Liang Y., Shi H. Appl. Surf. Sci. 264, 399-403 (2013).

35. Petroff Y., Yu P. Y, Shen Y. R. Phys. Rev. B. 12, 2488-2495 (1975).

36. Debbichi L., Marco de Lucas M.C., Pierson J.F., Kruger P. J. Phys. Chem. C. 116, 10232-10237 (2012).

37. Balamurugan B., Mehta B.R., Avashti D.K., Singh F., Arora A. K., Rajalakshmi M., Raghavan G., Tyagi A.K., Shivaprasad S.M. J. Appl. Phys. 92, 3304-3310 (2002).

38. Platzman I., Brener R., Haick H., Tonnenbaum R. J. Phys. Chem. C. 112, 1101-1108 (2008).

39. Mcintyre N.S., Cook M.G. Anal. Chem. 47, 2208-2213 (1975).

40. Chavez K.L., Hess D.W. J. Electrochem. Soc. 148, G640-G643 (2001).

41. Saikova S., Vorobyev S., Likhatski M., Romanchenko A., Erenburg S., Trubina S., Mikhlin Y. Appl. Surf. Sci. 258, 8214-8221 (2012).

42. Ramanathant S., Oyama S. T. J. Phys. Chem. 99, 16365-16372 (1995).

43. Thermo Scientific XPS knowledge base (http://xpssimplified.com/elements/carbon.php ,last accessed on 10 August 2016).

44. Wang M., Gu X.-K., Su H.-Y., Lu J.-M, Ma J.-P., Yu M., Zhang Z., Wang F. J. Catal. 330, 458-464 (2015).

45. Gu A., Wang G., Zhang X., Fang B. Bull. Mater. Sci., 33(1), 17-20 (2010).

46. Song M.-J., Hwang S.W., Whang D. Talanta 80, 1648-1652 (2010).

47. Wang H., Pan Q., Zhao J., Yin G., Zuo P. J. Power Sources 167, $206-211$ (2007).

48. Zhao G., Zhang L., Wang B., Sun K. Electrocbim. Acta 184, 117-123, (2015). 
49. Chen L., Shet S., Tang H., Wang H., Deutsch T., Yan Y., Turner J., Al-Jassim M. J. Mater. Chem. 20, 69626967 (2010).

50. Liu H., Hu Z., Hu R., Liu B., Ruan H., Zhang L., Xiao W. Int. J. Elctrochem. Sci. 11, 2756-2761 (2016).

51. Pires C. A., dos Santos A. C. C., Jordao E. Bra₹. J. Chem. Eng. 32, 04, 837-848 (2015).

52. Santos A., Yustos P., Quintanilla A., Rodriguez S., Garcia-Ochoa F. Appl. Catal., B 39, 97-113 (2002).

53. Alejandre A., Medina F., Fortuny A., Salagre P., Sueiras J.E. Appl. Catal., B 16, $53-67$ (1998).

54. Kurioka H., Uyama H., Kobayashi Sh. Polym. J. 30, 526-529 (1998).

55. Kobayashi Sh., Higashimura H. Prog. Polym. Sci. 28, 1015-1048 (2003).

56. Pintar A., Levec J. J. Catal. 135, 345-357 (1992).

\section{Acknowledgements}

The work was partially financed by the project Suplacat (Academy of Finland). R. Hajimammadov acknowledges the funding received from the University of Oulu Graduate School, Advanced Materials Doctoral Programme. Authors thank Sami Saukko with the assistance in XRD measurements. Santtu Heinilehto and Päivi Joensuu are acknowledged for performing XPS and GC-MS measurements, respectively.

\section{Other information}

K.K., and R.H. conceived the experiments. R.H. conducted the $\mathrm{Cu}$ nanowire growth and oxidation experiments, electrical, XRD, Raman and SEM measurements. AFM measurements were carried out by J.-M.O. under the supervision of G.S.L. The experimental data were discussed and interpreted by R.H. and K.K. with the help of M.M. and Z.C. The manuscript was written by R.H. with the help of K.K., M.M. and Z.C. All authors reviewed the manuscript. The Authors declare no competing financial interests. 